\title{
Sivers and $\cos 2 \phi$ asymmetries in semi-inclusive deep inelastic scattering in light-front holographic model
}

\author{
Tanmay Maji, ${ }^{1}$ Dipankar Chakrabarti, ${ }^{1}$ and Asmita Mukherjee ${ }^{2}$ \\ ${ }^{1}$ Department of Physics, Indian Institute of Technology Kanpur, Kanpur 208016, India \\ ${ }^{2}$ Department of Physics, Indian Institute of Technology Bombay, Powai, Mumbai 400076, India
}

(Received 20 November 2017; published 25 January 2018)

\begin{abstract}
The spin asymmetries in SIDIS associated with $T$-odd TMDs are presented in a light-front quark-diquark model of a proton. To incorporate the effects of the final-state interaction, the light front wave functions are modified to have a phase factor which is essential to have Sivers or Boer-Mulders functions. The Sivers and Boer-Mulder asymmetries are compared with HERMES and COMPASS data.
\end{abstract}

DOI: 10.1103/PhysRevD.97.014016

\section{INTRODUCTION}

Transverse momentum dependent parton distributions (TMDs) [1,2] are important to understand the single spin asymmetries observed experimentally for a long time $[3,4]$. They give a three-dimensional picture of the nucleons, together with the generalized parton distributions (GPDs) and represent nontrivial and nonperturbative correlations between the intrinsic transverse momentum of the partons (quarks and gluons) and the spin of the nucleon. Of particular interest are the Sivers function [5] and the Boer-Mulders function [6]. At the parton level, the Sivers function represents the coupling of the intrinsic transverse momentum of the partons to the transverse spin of the target. It quantifies the distribution of unpolarized quarks inside a transversely polarized target. The Sivers effect is particularly interesting as it is sensitive to a phase interference in the amplitudes [7] related to the gauge invariance of the underlying QCD interaction [8-11]. Namely, the Sivers function is nonzero only if it takes into account the gluonic initial and final-state interactions (FSIs). Such interactions are process dependent. The general structure of the process dependence may be quite complicated; however, the Sivers function for semiinclusive deep inelastic scattering (SIDIS) is expected to be negative to the Sivers function in the Drell-Yan (DY) process $[9,10]$. The Sivers effect produces azimuthal asymmetry in SIDIS that has been measured in experiment by HERMES, COMPASS and JLab [12-15]. The BoerMulders effect in the Drell-Yan process has been

Published by the American Physical Society under the terms of the Creative Commons Attribution 4.0 International license. Further distribution of this work must maintain attribution to the author(s) and the published article's title, journal citation, and DOI. Funded by SCOAP . investigated in Fermilab and preliminary results are available [16]. There are also recent data from $\mathrm{W}$ production at the RHIC [17]. Another TMD that has gathered considerable interest recently is the Boer-Mulders function [6], which gives the distribution of transversely polarized quarks in an unpolarized nucleon. This measures the spin-orbit correlations of quarks. The Boer-Mulders effect produces a measurable $\cos 2 \phi$ azimuthal asymmetry in SIDIS. Like the Sivers function, the Boer-Mulders function is also process dependent, due to the initial and final-state interactions.

There have been a lot of phenomenological studies on the Sivers as well as Boer-Mulders function (see, for example, [18-27]). A lattice calculation is presented in [28]. Extraction of the TMD parton distribution functions from experimental data usually relies on the following assumptions [24]: (i) factorization of the $x$ dependent part of the TMD from the $k_{\perp}^{2}$ dependent part, (ii) the $k_{\perp}$ dependent part is a Gaussian, and (iii) in the extraction of the Boer-Mulders function, one usually assumes that it is proportional to the Sivers function. The TMD functions are parametrized in terms of several parameters including the average transverse momenta $\left\langle k_{\perp}^{2}\right\rangle$ of the partons. This introduces an uncertainty as the experimental values of $\left\langle k_{\perp}^{2}\right\rangle$ are still not convergent: $\left\langle k_{\perp}^{2}\right\rangle \approx 0.25 \mathrm{GeV}^{2}$ from old EMC data and FNAL SIDIS data, whereas the value is $0.18 \mathrm{GeV}^{2}$ derived from HERMES data and this is the value that has been used in the extraction of the Boer-Mulders function. Analysis using more recent data suggests quite different value: $\left\langle k_{\perp}^{2}\right\rangle \approx 0.57 \mathrm{GeV}^{2}$ (HERMES) and $\left\langle k_{\perp}^{2}\right\rangle \approx 0.61 \mathrm{GeV}^{2}$ (COMPASS). A recent extraction [25] of the Sivers function, however, does not use any of these values of $\left\langle k_{\perp}^{2}\right\rangle$ as a parameter, but still it uses the factorization between the $x$ dependence and $k_{\perp}$ dependence. The current state of the art can be summarized by saying that the present data are 
insufficient to confirm the change of sign of the Sivers function between the SIDIS and DY processes, although there is a hint of such a sign change from $W^{-}$ production data at the RHIC [24].

The Sivers and Boer-Mulders TMDs have also been investigated in various phenomenological models [27,29-34]. In fact the first model calculation of the Sivers asymmetry in [7] showed the importance of the phase difference of the overlapping amplitudes to get a nonzero asymmetry. Model studies are also interesting to understand various relations between the TMDs and GPDs. An intuitive explanation of the Sivers effect was developed in [35] in a model-dependent way. The average transverse momentum of an unpolarized quark in a transversely polarized nucleon generated due to the Sivers effect is related to the distortion in impact parameter space through a lensing function, which is the effect of final-state interaction. This relation is found to hold in spectator-type models to the lowest nontrivial order, although expected to break down when higher order effects are taken into account. This relation is not expected to hold in models where the so-called lensing function does not factor out from the GPD in impart parameter space. This relation shows the connection of the Sivers function with the orbital angular momentum of the quarks although depending on the model. A similar model-dependent relation is derived in [36] between the Boer-Mulders function, which is related to the first derivative of the chiral odd GPDs $\mathcal{E}_{T}+2 \tilde{H}_{T}$ in the impact parameter space though the lensing function. The Sivers function and Boer-Mulders function are time-reversal $(\mathrm{T})$ odd functions whereas the GPDs above are $\mathrm{T}$ even, and no model-independent relation can be derived connecting them. Of course, GPDs and TMDs can be connected through different limits of the generalized transverse momentum dependent parton distribution functions. The motivation of the present work is to calculate the Sivers and Boer-Mulders function using a recently developed quark-diquark model light-front wave function of the proton based on light-front holography, calculate the asymmetries to compare with the data and investigate to what extent the model-dependent relations hold.

The light-front quark-diquark model [37] is briefly discussed in the next section. The model has been used to investigate Wigner distributions, GPDs and T-even TMDs [38-40]. The model is also found to predict the single-spin asymmetries described by T-even TMDs (Collins asymmetries) quite accurately at different experimental scales [41]. In this work, the model has been extended to incorporate the FSI into the light-front wave functions (LFWFs). The FSI generates a phase in the wave function which is responsible for nonzero T-odd TMDs, i.e., Sivers and Boer-Mulders functions and hence the spin asymmetries associated with them. The spin asymmetries evaluated in this model are compared with the experimental data using the QCD evolution prescribed by Abyat and Rogers [42].

\section{LIGHT-FRONT QUARK-DIQUARK MODEL FOR NUCLEON}

In the light-front quark-diquark model, the proton state is written in a linear combination of a quark-diquark state with the scalar and axial-vector diquark, considering the spin-flavor $S U(4)$ structure $[37,43,44]$ as

$$
|P ; \pm\rangle=C_{S}\left|u S^{0}\right\rangle^{ \pm}+C_{V}\left|u A^{0}\right\rangle^{ \pm}+C_{V V}\left|d A^{1}\right\rangle^{ \pm}
$$

where $C_{S}, C_{V}$ and $C_{V V}$ are the coefficient of the isoscalarscalar diquark singlet state $\left|u S^{0}\right\rangle$, isoscalar-axial vector diquark state $\left|u A^{0}\right\rangle$ and isovector-axial vector diquark state $\left|d A^{1}\right\rangle$ respectively. $S$ and $A$ represent the scalar and axialvector diquark with isospin at their superscript. Under the isospin symmetry, the neutron state is defined by the above formula with $u \leftrightarrow d$.

The light-cone coordinated $x^{ \pm}=x^{0} \pm x^{3}$. We choose a frame where the incoming proton does not have transverse momentum i.e. $P \equiv\left(P^{+}, \frac{M^{2}}{P^{+}}, \mathbf{0}_{\perp}\right)$. However, the struck quark and diquark have equal and opposite transverse momentum: $p \equiv\left(x P^{+}, \frac{p^{2}+\left|\mathbf{p}_{\perp}\right|^{2}}{x P^{+}}, \mathbf{p}_{\perp}\right)$ and $P_{X} \equiv\left((1-x) P^{+}\right.$, $\left.P_{X}^{-},-\mathbf{p}_{\perp}\right)$. Here $x=p^{+} / P^{+}$is the longitudinal momentum fraction carried by the struck quark. Detail kinematics of $\gamma^{*} P \rightarrow q(q q)$ are given for the tree level and final-stateinteraction diagram in Fig. 1.

The two-particle Fock-state expansion for $J^{z}= \pm 1 / 2$ for the spin-0 diquark state is given by

$$
|u S\rangle^{ \pm}=\int \frac{d x d^{2} \mathbf{p}_{\perp}}{2(2 \pi)^{3} \sqrt{x(1-x)}}\left[\psi_{+}^{ \pm(u)}\left(x, \mathbf{p}_{\perp}\right)\left|+\frac{1}{2} s ; x P^{+}, \mathbf{p}_{\perp}\right\rangle+\psi_{-}^{ \pm(u)}\left(x, \mathbf{p}_{\perp}\right)\left|-\frac{1}{2} s ; x P^{+}, \mathbf{p}_{\perp}\right\rangle\right]
$$

where $\left|\lambda_{q} \lambda_{S} ; x P^{+}, \mathbf{p}_{\perp}\right\rangle$ is the two-particle state having struck a quark of helicity $\lambda_{q}$ and a scalar diquark having helicity $\lambda_{S}=s$ (spin-0 singlet diquark helicity is denoted by s to distinguish from the triplet diquark). The state with spin-1 diquark is given as [45] 


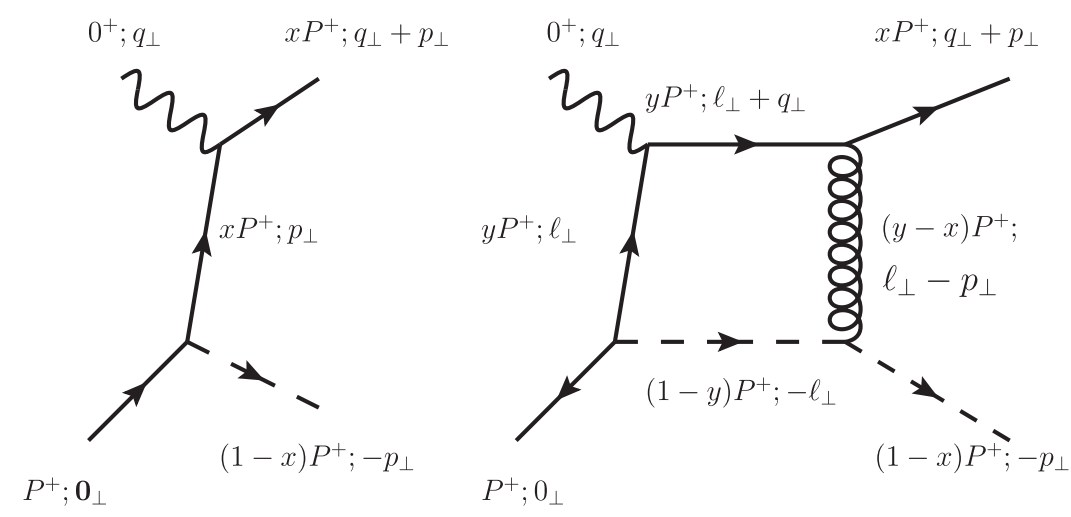

(a)

(b)

FIG. 1. Left: tree level diagram. Right: FSI diagram for $\gamma^{*} P \rightarrow q(q q)$.

$$
\begin{aligned}
|\nu A\rangle^{ \pm}= & \int \frac{d x d^{2} \mathbf{p}_{\perp}}{2(2 \pi)^{3} \sqrt{x(1-x)}}\left[\psi_{++}^{ \pm(\nu)}\left(x, \mathbf{p}_{\perp}\right)\left|+\frac{1}{2}+1 ; x P^{+}, \mathbf{p}_{\perp}\right\rangle\right. \\
& +\psi_{-+}^{ \pm(\nu)}\left(x, \mathbf{p}_{\perp}\right)\left|-\frac{1}{2}+1 ; x P^{+}, \mathbf{p}_{\perp}\right\rangle+\psi_{+0}^{ \pm(\nu)}\left(x, \mathbf{p}_{\perp}\right)\left|+\frac{1}{2} 0 ; x P^{+}, \mathbf{p}_{\perp}\right\rangle \\
& +\psi_{-0}^{ \pm(\nu)}\left(x, \mathbf{p}_{\perp}\right)\left|-\frac{1}{2} 0 ; x P^{+}, \mathbf{p}_{\perp}\right\rangle+\psi_{+-}^{ \pm(\nu)}\left(x, \mathbf{p}_{\perp}\right)\left|+\frac{1}{2}-1 ; x P^{+}, \mathbf{p}_{\perp}\right\rangle \\
& \left.+\psi_{--}^{ \pm(\nu)}\left(x, \mathbf{p}_{\perp}\right)\left|-\frac{1}{2}-1 ; x P^{+}, \mathbf{p}_{\perp}\right\rangle\right],
\end{aligned}
$$

where $\nu$ is the flavor index for $u$ and $d$ and $\left|\lambda_{q} \lambda_{D} ; x P^{+}, \mathbf{p}_{\perp}\right\rangle$ represents a two-particle state with a quark of helicity $\lambda_{q}= \pm \frac{1}{2}$ and an axial-vector diquark of helicity $\lambda_{D}= \pm 1,0$ (triplet).

\section{FINAL-STATE INTERACTION AND T-ODD TMDS}

The final-state interaction [46] produces a nontrivial phase in the amplitude and gives nonvanishing T-odd TMDs along with the T-even TMDs. There are two T-odd TMDs, $f_{1 T}^{\perp \nu}\left(x, \mathbf{p}_{\perp}^{2}\right)$ (Sivers function) and $h_{1}^{\perp \nu}\left(x, \mathbf{p}_{\perp}^{2}\right)$ (Boer-Mulders function), at the leading twist. The contribution of FSI is incorporated in the wave functions [47] and the wave functions are modified with spin dependent complex phases as

(i) for the scalar diquark

$$
\begin{aligned}
& \psi_{+}^{+(u)}\left(x, \mathbf{p}_{\perp}\right)=N_{S}\left[1+i \frac{e_{1} e_{2}}{8 \pi}\left(\mathbf{p}_{\perp}^{2}+B\right) g_{1}\right] \varphi_{1}^{(u)}\left(x, \mathbf{p}_{\perp}\right), \\
& \psi_{-}^{+(u)}\left(x, \mathbf{p}_{\perp}\right)=N_{S}\left(-\frac{p^{1}+i p^{2}}{x M}\right)\left[1+i \frac{e_{1} e_{2}}{8 \pi}\left(\mathbf{p}_{\perp}^{2}+B\right) g_{2}\right] \varphi_{2}^{(u)}\left(x, \mathbf{p}_{\perp}\right), \\
& \psi_{+}^{-(u)}\left(x, \mathbf{p}_{\perp}\right)=N_{S}\left(\frac{p^{1}-i p^{2}}{x M}\right)\left[1+i \frac{e_{1} e_{2}}{8 \pi}\left(\mathbf{p}_{\perp}^{2}+B\right) g_{2}\right] \varphi_{2}^{(u)}\left(x, \mathbf{p}_{\perp}\right), \\
& \psi_{-}^{-(u)}\left(x, \mathbf{p}_{\perp}\right)=N_{S}\left[1+i \frac{e_{1} e_{2}}{8 \pi}\left(\mathbf{p}_{\perp}^{2}+B\right) g_{1}\right] \varphi_{1}^{(u)}\left(x, \mathbf{p}_{\perp}\right),
\end{aligned}
$$


(ii) for the axial-vector diquark (for $J= \pm 1 / 2$ )

$$
\begin{aligned}
& \psi_{++}^{+(\nu)}\left(x, \mathbf{p}_{\perp}\right)=N_{1}^{(\nu)} \sqrt{\frac{2}{3}}\left(\frac{p^{1}-i p^{2}}{x M}\right)\left[1+i \frac{e_{1} e_{2}}{8 \pi}\left(\mathbf{p}_{\perp}^{2}+B\right) g_{2}\right] \varphi_{2}^{(\nu)}\left(x, \mathbf{p}_{\perp}\right), \\
& \psi_{-+}^{+(\nu)}\left(x, \mathbf{p}_{\perp}\right)=N_{1}^{(\nu)} \sqrt{\frac{2}{3}}\left[1+i \frac{e_{1} e_{2}}{8 \pi}\left(\mathbf{p}_{\perp}^{2}+B\right) g_{1}\right] \varphi_{1}^{(\nu)}\left(x, \mathbf{p}_{\perp}\right), \\
& \psi_{+0}^{+(\nu)}\left(x, \mathbf{p}_{\perp}\right)=-N_{0}^{(\nu)} \sqrt{\frac{1}{3}}\left[1+i \frac{e_{1} e_{2}}{8 \pi}\left(\mathbf{p}_{\perp}^{2}+B\right) g_{1}\right] \varphi_{1}^{(\nu)}\left(x, \mathbf{p}_{\perp}\right), \\
& \psi_{-0}^{+(\nu)}\left(x, \mathbf{p}_{\perp}\right)=N_{0}^{(\nu)} \sqrt{\frac{1}{3}}\left(\frac{p^{1}+i p^{2}}{x M}\right)\left[1+i \frac{e_{1} e_{2}}{8 \pi}\left(\mathbf{p}_{\perp}^{2}+B\right) g_{2}\right] \varphi_{2}^{(\nu)}\left(x, \mathbf{p}_{\perp}\right), \\
& \psi_{+-}^{+(\nu)}\left(x, \mathbf{p}_{\perp}\right)=0, \\
& \psi_{--}^{+(\nu)}\left(x, \mathbf{p}_{\perp}\right)=0,
\end{aligned}
$$

and for $J=-1 / 2$

$$
\begin{aligned}
& \psi_{++}^{-(\nu)}\left(x, \mathbf{p}_{\perp}\right)=0, \\
& \psi_{-+}^{-(\nu)}\left(x, \mathbf{p}_{\perp}\right)=0, \\
& \psi_{+0}^{-(\nu)}\left(x, \mathbf{p}_{\perp}\right)=N_{0}^{(\nu)} \sqrt{\frac{1}{3}}\left(\frac{p^{1}-i p^{2}}{x M}\right)\left[1+i \frac{e_{1} e_{2}}{8 \pi}\left(\mathbf{p}_{\perp}^{2}+B\right) g_{2}\right] \varphi_{2}^{(\nu)}\left(x, \mathbf{p}_{\perp}\right), \\
& \psi_{-0}^{-(\nu)}\left(x, \mathbf{p}_{\perp}\right)=N_{0}^{(\nu)} \sqrt{\frac{1}{3}}\left[1+i \frac{e_{1} e_{2}}{8 \pi}\left(\mathbf{p}_{\perp}^{2}+B\right) g_{1}\right] \varphi_{1}^{(\nu)}\left(x, \mathbf{p}_{\perp}\right), \\
& \psi_{+-}^{-(\nu)}\left(x, \mathbf{p}_{\perp}\right)=-N_{1}^{(\nu)} \sqrt{\frac{2}{3}}\left[1+i \frac{e_{1} e_{2}}{8 \pi}\left(\mathbf{p}_{\perp}^{2}+B\right) g_{1}\right] \varphi_{1}^{(\nu)}\left(x, \mathbf{p}_{\perp}\right), \\
& \psi_{--}^{-(\nu)}\left(x, \mathbf{p}_{\perp}\right)=N_{1}^{(\nu)} \sqrt{\frac{2}{3}}\left(\frac{p^{1}+i p^{2}}{x M}\right)\left[1+i \frac{e_{1} e_{2}}{8 \pi}\left(\mathbf{p}_{\perp}^{2}+B\right) g_{2}\right] \varphi_{2}^{(\nu)}\left(x, \mathbf{p}_{\perp}\right),
\end{aligned}
$$

where

$$
\begin{aligned}
g_{1} & =\int_{0}^{1} d \alpha \frac{-1}{\alpha(1-\alpha) \mathbf{p}_{\perp}^{2}+\alpha m_{g}^{2}+(1-\alpha) B}, \\
g_{2} & =\int_{0}^{1} d \alpha \frac{-\alpha}{\alpha(1-\alpha) \mathbf{p}_{\perp}^{2}+\alpha m_{g}^{2}+(1-\alpha) B}
\end{aligned}
$$

and,

$$
B=x(1-x)\left(-M^{2}+\frac{m_{q}^{2}}{x}+\frac{m_{D}^{2}}{1-x}\right) .
$$

$M, m_{q}, m_{D}$ and $m_{g}$ are the mass of the proton, struck quark, diquark and gluon respectively. We take $m_{g}=0$ at the end of the calculations. $N_{S}, N_{0}^{(\nu)}$ and $N_{1}^{(\nu)}$ are the normalization constants.

The LFWFs $\varphi_{i}^{(\nu)}\left(x, \mathbf{p}_{\perp}\right)$ are the modified form of the soft-wall AdS/QCD prediction as [48]

$$
\varphi_{i}^{(\nu)}\left(x, \mathbf{p}_{\perp}\right)=\frac{4 \pi}{\kappa} \sqrt{\frac{\log (1 / x)}{1-x}} x^{a_{i}^{\nu}}(1-x)^{b_{i}^{\nu}} \exp \left[-\delta^{\nu} \frac{\mathbf{p}_{\perp}^{2}}{2 \kappa^{2}} \frac{\log (1 / x)}{(1-x)^{2}}\right],
$$

introducing the parameters $a_{i}^{\nu}, b_{i}^{\mu}$ and $\delta^{\nu}$. The wave functions $\varphi_{i}^{\nu}(i=1,2)$ reduce to the AdS/QCD prediction [49] for the parameters $a_{i}^{\nu}=b_{i}^{\nu}=0$ and $\delta^{\nu}=1.0$. We use the AdS/QCD scale parameter $\kappa=0.4 \mathrm{GeV}$ as determined in [50] and the quarks are assumed to be massless. 
The unintegrated quark-quark correlator for polarized SIDIS is defined as

$$
\begin{aligned}
\Phi^{\nu[\Gamma]}\left(x, \mathbf{p}_{\perp} ; S\right)= & \frac{1}{2} \int \frac{d z^{-} d^{2} z_{T}}{2(2 \pi)^{3}} \\
& \times e^{i p . z}\left\langle P ; S\left|\bar{\psi}^{\nu}(0) \Gamma \mathcal{W}_{[0, z]} \psi^{\nu}(z)\right| P ; S\right\rangle,
\end{aligned}
$$

with a flavor $\nu$. The summations over the color indices of quarks are implied. $\left.\mathcal{W}_{[} 0, z\right]$ is the Wilson line, the effect of which is incorporated in the LFWFs in terms of FSI. Here, $p$ is the momentum of the struck quark inside the nucleon of momentum $\mathrm{P}$, spin $\mathrm{S}$ and $x\left(x=p^{+} / P^{+}\right)$is the longitudinal momentum fraction carried by the struck quark.
We choose the light-cone gauge $A^{+}=0$. The nucleon with helicity $\lambda_{N}$ has spin components $S^{+}=\lambda_{N} \frac{P^{+}}{M}, S^{-}=\lambda_{N} \frac{P^{-}}{M}$, and $S_{T}$. At leading twist, the T-odd TMDs are defined as

$$
\begin{gathered}
\Phi^{\nu\left[\gamma^{+}\right]}\left(x, \mathbf{p}_{\perp} ; S\right)=\cdots-\frac{\epsilon_{T}^{i j} p_{\perp}^{i} S_{T}^{j}}{M} f_{1 T}^{\perp \nu}\left(x, \mathbf{p}_{\perp}^{2}\right), \\
\Phi^{\nu\left[i \sigma^{j+} \gamma^{5}\right]}\left(x, \mathbf{p}_{\perp} ; S\right)=\cdots+\frac{\epsilon_{T}^{i j} p_{\perp}^{i}}{M} h_{1}^{\perp \nu}\left(x, \mathbf{p}_{\perp}^{2}\right),
\end{gathered}
$$

where the ellipses indicate the terms involving T-even TMDs.

Using Eq. (1) in (11) the correlators for the transversely polarized proton are written in terms of overlap representations as

$$
\begin{aligned}
\Phi^{\nu\left[\gamma^{+}\right]}\left(x, \mathbf{p}_{\perp} ; \uparrow\right)= & \frac{1}{2}\left[C_{S}^{2} \frac{1}{16 \pi^{3}} \sum_{\lambda_{q}} \sum_{\lambda_{N}} \sum_{\lambda_{N}^{\prime}} \psi_{\lambda_{q}}^{\lambda_{N}^{\dagger}}\left(x, \mathbf{p}_{\perp}\right) \psi_{\lambda_{q}}^{\lambda_{N}^{\prime}}\left(x, \mathbf{p}_{\perp}\right)\right]^{\nu} \\
& +\frac{1}{2}\left[C_{A}^{2} \frac{1}{16 \pi^{3}} \sum_{\lambda_{q}} \sum_{\lambda_{D}} \sum_{\lambda_{N}} \sum_{\lambda_{N}^{\prime}} \psi_{\lambda_{q} \lambda_{D}}^{\lambda_{N} \dagger}\left(x, \mathbf{p}_{\perp}\right) \psi_{\lambda_{q} \lambda_{D}}^{\lambda_{N}^{\prime}}\left(x, \mathbf{p}_{\perp}\right)\right]^{\nu}, \\
\Phi^{\nu\left[i \sigma^{1+} \gamma^{5}\right]}\left(x, \mathbf{p}_{\perp} ; \uparrow\right)= & \frac{1}{2}\left[C_{S}^{2} \frac{1}{16 \pi^{3}} \sum_{\lambda_{q}} \sum_{\lambda_{q}^{\prime}} \sum_{\lambda_{N}} \psi_{\lambda_{q}}^{\lambda_{N} \dagger}\left(x, \mathbf{p}_{\perp}\right) \psi_{\lambda_{q}^{\prime}}^{\lambda_{N}}\left(x, \mathbf{p}_{\perp}\right)\right]^{\nu} \\
& +\frac{1}{2}\left[C_{A}^{2} \frac{1}{16 \pi^{3}} \sum_{\lambda_{q}} \sum_{\lambda_{q}^{\prime}} \sum_{\lambda_{D}} \sum_{\lambda_{N}} \psi_{\lambda_{q} \lambda_{D}}^{\lambda_{N} \dagger}\left(x, \mathbf{p}_{\perp}\right) \psi_{\lambda^{\prime} \lambda_{D}}^{\lambda_{N}}\left(x, \mathbf{p}_{\perp}\right)\right]^{\nu},
\end{aligned}
$$

where $\lambda_{q}, \lambda_{D}= \pm$ represent the helicity of the quark and diquark respectively. In Eq. (17) the quark polarization is taken along the $x$-axis, $j=1$. The first term in the right-hand side is for the scalar diquark and the second term corresponds to the vector diquark. $N_{i}^{\prime} s$ are the normalization constants and $N_{S}=2.0191, N_{0}^{(u)}=3.2050, N_{0}^{(d)}=$ 5.9423, $N_{1}^{(u)}=0.9895, N_{d}^{(d)}=1.1616$. Note, the first terms in the right-hand side of Eqs. (14) and (15) become 0 for d quark as $N_{S}^{d}=0$ in the scalar wave functions. $C_{A}^{2}$ in the second term stands for the coefficients $C_{V}^{2}$ and $C_{V V}^{2}$ for the $\mathrm{u}$ quark and d quark respectively. The coefficients are fixed by the quark counting rules: $C_{S}^{2}=1.3872, C_{V}^{2}=$ 0.6128 and $C_{V V}^{2}=1$. The details of the determination of the coefficients $C_{j}^{\prime} s$ and normalization constants $N_{i}^{\prime} s$ are discussed in [37]. Comparing Eqs. (12) and (13) with Eqs. (14) and (15) the Sivers function $f_{1 T}^{\perp \nu}\left(x, \mathbf{p}_{\perp}^{2}\right)$ and BoerMulders functions can be written in the light-front quarkdiquark model (LFQDM) as

$$
\begin{gathered}
f_{1 T}^{\perp \nu}\left(x, \mathbf{p}_{\perp}^{2}\right)=\left(C_{S}^{2} N_{S}^{\nu 2}-C_{A}^{2} \frac{1}{3} N_{0}^{\nu 2}\right) f^{\nu}\left(x, \mathbf{p}_{\perp}^{2}\right), \\
h_{1}^{\perp \nu}\left(x, \mathbf{p}_{\perp}^{2}\right)=\left(C_{S}^{2} N_{S}^{\nu 2}+C_{A}^{2}\left(\frac{1}{3} N_{0}^{\nu 2}+\frac{2}{3} N_{1}^{\nu 2}\right)\right) f^{\nu}\left(x, \mathbf{p}_{\perp}^{2}\right),
\end{gathered}
$$

where

$$
\begin{aligned}
f^{\nu}\left(x, \mathbf{p}_{\perp}^{2}\right)= & -C_{F} \alpha_{s}\left[\mathbf{p}_{\perp}^{2}+x(1-x)\left(-M^{2}+\frac{m_{D}^{2}}{1-x}+\frac{m_{q}^{2}}{x}\right)\right] \frac{1}{\mathbf{p}_{\perp}^{2}} \\
& \times \ln \left[1+\frac{\mathbf{p}_{\perp}^{2}}{x(1-x)\left(-M^{2}+\frac{m_{D}^{2}}{1-x}+\frac{m_{q}^{2}}{x}\right)}\right] \\
& \times \frac{\ln (1 / x)}{\pi \kappa^{2}} x^{a_{1}^{\nu}+a_{2}^{\nu}-1}(1-x)^{b_{1}^{\nu}+b_{2}^{\nu}-1} \exp \left[-\delta^{\nu} \frac{\mathbf{p}_{\perp}^{2} \ln (1 / x)}{\kappa^{2}(1-x)^{2}}\right],
\end{aligned}
$$



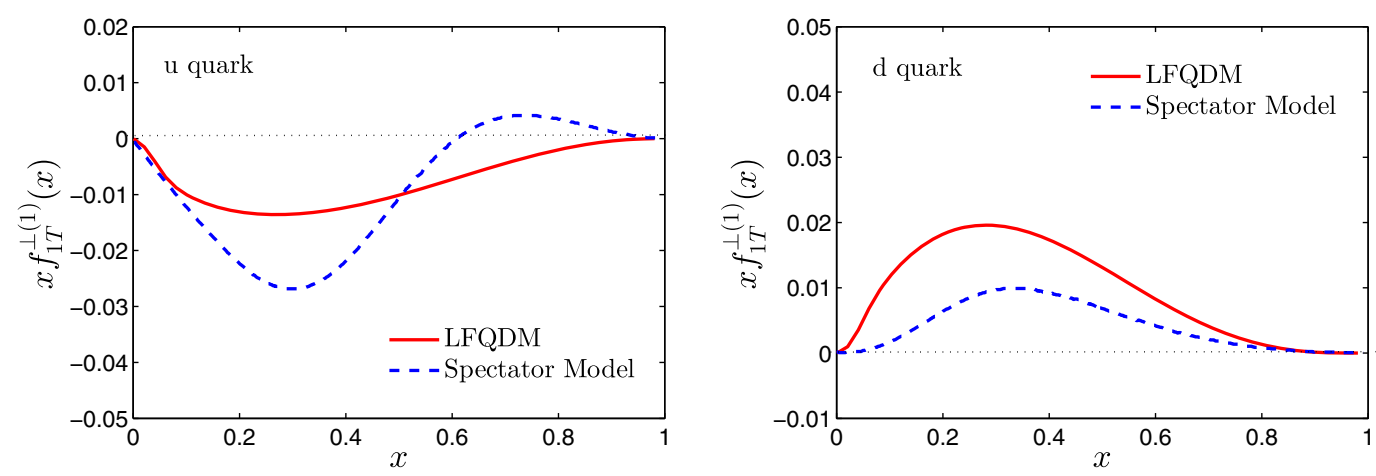

FIG. 2. $x f_{1 T}^{\perp(1)}(x)$ are for $u$ and $d$ quarks at initial scale $\mu_{0}=0.8 \mathrm{GeV}$. Red continuous lines represent the model result in LFQDM and the blue dashed line represents the result in the spectator model [44].

with struck quark mass $m_{q}$ and diquark mass $m_{D}$. In the finalstate interaction, gluon exchange strength $\frac{e_{1} e_{2}}{4 \pi} \rightarrow-C_{F} \alpha_{s}$. Here the $e_{1}$ and $e_{2}$ are the color charge of the struck quark and diquark respectively. The values of the parameters $a_{i}^{\nu}, b_{i}^{\nu}$ $(i=1,2)$ and $\delta^{\nu}$ are given in [39].

The moment of the Sivers functions, defined as

$$
f_{1 T}^{\perp(1)}(x)=\int d^{2} p_{\perp} \frac{p_{\perp}^{2}}{2 M^{2}} f_{1 T}^{\perp}\left(x, \mathbf{p}_{\perp}^{2}\right)
$$

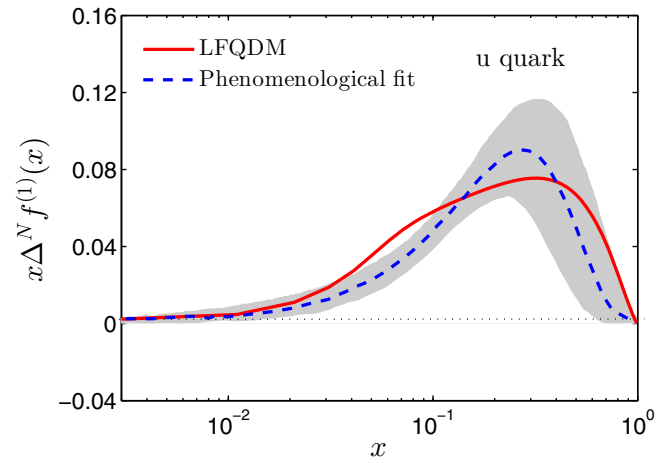

is shown in Fig. 2 at the initial scale and compared with the spectator model [44]. Our model result for the u quark does not have any positive peak like the spectator model. The scale evolution of the distributions is not included in the spectator model.

According to the Burkardt sum rule [51], the net transverse Sivers momentum when summed over all the constituents is 0 . In the quark-diquark model, the constituents are quarks $(q)$ and diquarks $(D)$ only and the statement can be written as

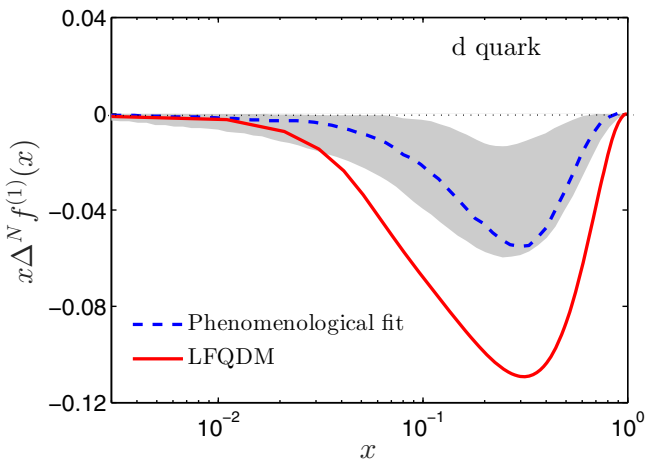

FIG. 3. $x \Delta^{N} f^{(1)}(x)$ are shown for $\mathrm{u}$ and $\mathrm{d}$ quarks at the scale $\mu=1 \mathrm{GeV}$. Our model result is shown in red continuous lines. Blue dashed lines represent the phenomenological extraction [53] from the best fit of the Sivers asymmetries measured by HERMES [14] and COMPASS [13,54] Collaborations.
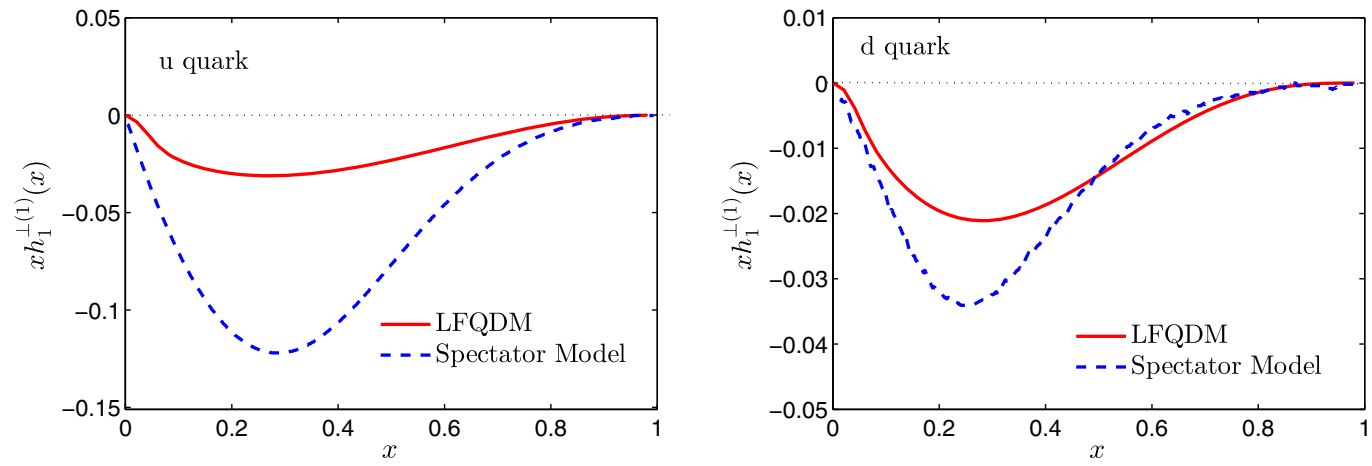

FIG. 4. $x h_{1}^{\perp(1)}(x)$ are shown for $\mathrm{u}$ and $\mathrm{d}$ quarks at initial scale $\mu_{0}=0.8 \mathrm{GeV}$. Red continuous lines represent the model result in LFQDM and the blue dashed line represents the result in the spectator model [44]. 
TABLE I. $\lambda^{\nu}$ of Eq. (27) for $u$ and d quarks are shown in our model and fitted data [26] of HERMES and COMPASS.

\begin{tabular}{lcc}
\hline \hline & $\lambda^{u}$ & $\lambda^{d}$ \\
\hline LFQDM & 2.29 & -1.08 \\
Phenomenological fit & $2.1 \pm 0.1$ & $-1.11 \pm 0.02$ \\
\hline \hline
\end{tabular}

$$
\sum_{i=q, D}\left\langle k_{\perp}^{i}\right\rangle=0
$$

The sum rule in terms of the Sivers function can be written as [19]

$$
\sum_{i=q, D} \int d x f_{1 T}^{\perp(1)}(x)=0 .
$$

In a scalar diquark model, it was shown [52] that the Sivers functions for the quark and diquark are related by

$$
f_{1 T}^{\perp D}\left(x, \mathbf{p}_{\perp}^{2}\right)=-f_{1 T}^{\perp q}\left(1-x, \mathbf{p}_{\perp}^{2}\right) .
$$

The same relation also holds in our model when averaged over the vector diquark polarizations and the Burkardt sum rule is satisfied.

In Fig. 3, the $x \Delta^{N} f^{(1)}(x)$ are presented at the scale $\mu=$ $1 \mathrm{GeV}$ and compared with the phenomenological fit from the HERMES and COMPASS data. The moment of the Sivers function $\Delta^{N} f^{(1)}(x)$ is defined as

$$
\Delta^{N} f^{(1)}(x)=\int d^{2} p_{\perp}\left(\frac{p_{\perp}}{4 M}\right) \Delta^{N} f_{\nu / P^{\uparrow}}\left(x, \mathbf{p}_{\perp}\right),
$$

where

$$
\Delta^{N} f_{\nu / P^{\uparrow}}\left(x, \mathbf{p}_{\perp}\right)=\left(-\frac{2 p_{\perp}}{M}\right) f_{1 T}^{\perp}\left(x, \mathbf{p}_{\perp}^{2}\right) .
$$

In Fig. 4, we show our model result for the moment of Boer-Mulder functions, defined as

$$
h_{1}^{\perp(1)}(x)=\int d^{2} p_{\perp} \frac{p_{\perp}^{2}}{2 M^{2}} h_{1}^{\perp}\left(x, \mathbf{p}_{\perp}^{2}\right),
$$

at the initial scale and compare with the spectator model. In this model, we observe

$$
\left|h_{1}^{\perp}\left(x, \mathbf{p}_{\perp}^{2}\right)\right|>\left|f_{1 T}^{\perp}\left(x, \mathbf{p}_{\perp}^{2}\right)\right| .
$$

From Eqs. (16) and (17), we can easily see that the BoerMulders function is proportional to the Sivers function. In fact, the Boer-Mulders function is parametrized [26] as

$$
h_{1}^{\perp \nu}\left(x, \mathbf{p}_{\perp}^{2}\right) \simeq \lambda^{\nu} f_{1 T}^{\perp \nu}\left(x, \mathbf{p}_{\perp}^{2}\right) .
$$

Table I shows our model result of $\lambda^{\nu}$ compared with the result of HERMES and COMPASS data fits [26] for $\cos 2 \phi$ asymmetry in SIDIS. The results indicate that BoerMulders functions are negative for both $\mathrm{u}$ and d quarks.

\section{SIVERS ASYMMETRY AND BOER-MULDERS ASYMMETRY}

The Sivers asymmetry correlates between transverse momentum of parton and transverse polarization of nucleon. In the SIDIS precesses, Sivers asymmetry can be extracted by incorporating the weight factor $\sin \left(\phi_{h}-\phi_{S}\right)$ as

$$
A_{U T}^{\sin \left(\phi_{h}-\phi_{S}\right)}=\frac{\int d \phi_{h} d \phi_{S}\left[d \sigma^{\ell P^{\uparrow} \rightarrow \ell^{\prime} h X}-d \sigma^{\ell P^{\natural \downarrow} \rightarrow \ell^{\prime} h X}\right] \sin \left(\phi_{h}-\phi_{S}\right)}{\int d \phi_{h} d \phi_{S}\left[d \sigma^{\ell P^{\uparrow} \rightarrow \ell^{\prime} h X}+d \sigma^{\ell P^{\downarrow} \rightarrow \ell^{\prime} h X}\right]}
$$

where $\uparrow, \downarrow$ at the superscript of $P$ represent the up and down transverse spin of the target proton. According to the QCD factorization scheme the SIDIS cross section for the one photon exchange process $\ell N \rightarrow \ell^{\prime} h X$ is written as

$$
\begin{aligned}
d \sigma^{\ell N \rightarrow \ell^{\prime} h X}= & \sum_{\nu} \hat{f}_{\nu / P}\left(x, \mathbf{p}_{\perp} ; Q^{2}\right) \otimes d \hat{\sigma}^{\ell q \rightarrow \ell q} \\
& \otimes \hat{D}_{h / \nu}\left(z, \mathbf{k}_{\perp} ; Q^{2}\right)
\end{aligned}
$$

where the second term represents the hard scattering part which is calculable in perturbative QCD. The soft part is factorized into TMDs, denoted by $\hat{f}_{\nu / P}\left(x, \mathbf{p}_{\perp} ; Q^{2}\right)$ and fragmentation functions (FF), denoted by $\hat{D}_{h / \nu}\left(z, \mathbf{k}_{\perp} ; Q^{2}\right)$. This scheme holds in the small $\mathbf{P}_{h \perp}$ and large $Q$ region,
$P_{h \perp}^{2} \simeq \Lambda_{\mathrm{OCD}}^{2} \ll Q^{2}$. The quark-gluon corrections and higher order perturbative QCD corrections become important at the large $\mathbf{P}_{h \perp}$ regime [44,55,56]. The TMD factorization is presented for the SIDIS and the DY processes in [57-61] and later on used in [42,53,62,63]. The kinematic variables are defined in the $\gamma^{*}-N$ center of mass frame as

$$
\begin{aligned}
& x=\frac{Q^{2}}{2(P . q)}=x_{B}, \\
& z=\frac{P . P_{h}}{P . q}=z_{h}, \\
& y=\frac{P . q}{P . \ell}=\frac{Q^{2}}{s x} .
\end{aligned}
$$


Bjorken variable $x_{B}=\frac{Q^{2}}{2 P . q}$ with $Q^{2}=-q^{2}$. The fractional energy transferred by the photon in the lab system is $y$ and the energy fraction carried by the produced hadron is $z=\mathbf{P}_{h}^{-} / k^{-}$. The transverse momentum of the fragmenting quark is denoted as $\mathbf{k}_{\perp}$. The momentum of the virtual photon $q \equiv\left(x_{B} P^{+}, \frac{Q^{2}}{x_{B} P^{+}}, \mathbf{0}_{\perp}\right)$ and of the incoming proton $P \equiv\left(P^{+}, \frac{M^{2}}{P^{+}}, \mathbf{0}_{\perp}\right)$. The struck quark have nonzero transverse momentum $\mathbf{p}_{\perp}$ with the momentum $p \equiv\left(x P^{+}, \frac{p^{2}+\left|\mathbf{p}_{\perp}\right|^{2}}{x P^{+}}, \mathbf{p}_{\perp}\right)$ and the diquark carries $p_{D} \equiv\left((1-x) P^{+}, \frac{p^{2}+\left|\mathbf{p}_{\perp}\right|^{2}}{(1-x) P^{+}},-\mathbf{p}_{\perp}\right)$. In this frame, the produced hadron has a finite transverse momentum $\mathbf{P}_{h \perp}$. At $\mathcal{O}\left(\mathbf{p}_{\perp} / Q\right)$, the relation between $\mathbf{p}_{\perp}, \quad \mathbf{k}_{\perp}$ and $\quad \mathbf{P}_{h \perp} \quad$ is given as $\quad \mathbf{k}_{\perp}=\mathbf{P}_{h \perp}-z \mathbf{p}_{\perp}$. The transverse momentum of the produced hadron makes an azimuthal angle $\phi_{h}$ with respect to the lepton plane and transverse spin $\left(S_{P}\right)$ of the proton has an azimuthal angle $\phi_{S}$. Then the SIDIS crosssection difference [64] in the numerator can be written as

$$
\begin{aligned}
\frac{d \sigma^{\ell P^{\uparrow} \rightarrow \ell^{\prime} h X}-d \sigma^{\ell P^{\downarrow} \rightarrow \ell^{\prime} h X}}{d x_{B} d y d z d^{2} \mathbf{P}_{h \perp} d \phi_{S}}= & \frac{2 \alpha^{2}}{s x y^{2}} 2\left[\frac{1+(1-y)^{2}}{2} \sin \left(\phi_{h}-\phi_{S}\right) F_{U T}^{\sin \left(\phi_{h}-\phi_{S}\right)}\right. \\
& +(1-y)\left(\sin \left(\phi_{h}+\phi_{S}\right) F_{U T}^{\sin \left(\phi_{h}+\phi_{S}\right)}+\sin \left(3 \phi_{h}-\phi_{S}\right) F_{U T}^{\sin \left(3 \phi_{h}-\phi_{S}\right)}\right) \\
& \left.+(2-y) \sqrt{(1-y)}\left(\sin \phi_{S} F_{U T}^{\sin \phi_{S}}+\sin \left(2 \phi_{h}-\phi_{S}\right) F_{U T}^{\sin \left(2 \phi_{h}-\phi_{S}\right)}\right)\right] .
\end{aligned}
$$

The weighted structure functions, $F_{S_{\ell} S}^{\mathcal{W}\left(\phi_{h}, \phi_{S}\right)}$, are defined as

$$
\begin{aligned}
F_{S_{\ell} S}^{\mathcal{W}\left(\phi_{h}, \phi_{S}\right)}= & \sum_{\nu} e_{\nu}^{2} \int d^{2} \mathbf{p}_{\perp} d^{2} \mathbf{k}_{\perp} \delta^{(2)} \\
& \times\left(\mathbf{P}_{h \perp}-z \mathbf{p}_{\perp}-\mathbf{k}_{\perp}\right) \mathcal{W}\left(\mathbf{p}_{\perp}, \mathbf{P}_{h \perp}\right) \\
& \times \hat{f}^{\nu}\left(x, \mathbf{p}_{\perp}\right) \hat{D}^{\nu}\left(z, \mathbf{k}_{\perp}\right),
\end{aligned}
$$

where $\hat{f}^{\nu}\left(x, \mathbf{p}_{\perp}\right)$ and $\hat{D}^{\nu}\left(z, \mathbf{k}_{\perp}\right)$ represent leading twist TMDs and FFs respectively. Integrating the numerator over $\phi_{h}$ and $\phi_{S}$, with a particular weight factor $\mathcal{W}\left(\phi_{h}, \phi_{S}\right)$, one can project out the corresponding structure function $F_{S_{e} S}^{\mathcal{W}\left(\phi_{h}, \phi_{S}\right)}$ and hence the particular asymmetry can be found. For example, the $\phi_{h}$ and $\phi_{S}$ integration with the weight factors $\sin \left(\phi_{h}-\phi_{S}\right)$ and $\cos \left(2 \phi_{h}\right)$ ends up with the Sivers asymmetry and Boer-Mulders asymmetry.

Similarly, the denominator can be written as

$$
\begin{aligned}
& \frac{d \sigma^{\ell P^{\uparrow} \rightarrow \ell^{\prime} h X}+d \sigma^{\ell P^{\downarrow} \rightarrow \ell^{\prime} h X}}{d x_{B} d y d z d^{2} \mathbf{P}_{h \perp} d \phi_{S}} \\
& =\frac{2 \alpha^{2}}{s x y^{2}} 2\left[\frac{1+(1-y)^{2}}{2} F_{U U}+(2-y) \sqrt{1-y} \cos \phi_{h} F_{U U}^{\cos \phi_{h}}\right. \\
& \left.\quad+(1-y) \cos 2 \phi_{h} F_{U U}^{\cos 2 \phi_{h}}\right]
\end{aligned}
$$

Thus Sivers asymmetry can be written in terms of structure functions [64] as

$$
\begin{aligned}
A_{U T}^{\sin \left(\phi_{h}-\phi_{S}\right)}\left(x, z, \mathbf{P}_{h \perp}, y\right) & =\frac{2 \pi^{2} \alpha^{2} \frac{1+(1-y)^{2}}{s x y^{2}} F_{U T}^{\sin \left(\phi_{h}-\phi_{S}\right)}\left(x, z, \mathbf{P}_{h \perp}\right)}{2 \pi^{2} \alpha^{2} \frac{1+(1-y)^{2}}{s x y^{2}} F_{U U}\left(x, z, \mathbf{P}_{h \perp}\right)} \\
& =\frac{2 \pi^{2} \alpha^{2} \frac{1+(1-y)^{2}}{s x y^{2}} \sum_{\nu} e_{\nu}^{2} \int d^{2} p_{\perp}\left\{\frac{-\hat{\mathbf{P}}_{h \perp} \cdot \mathbf{p}_{\perp}}{M}\right\} f_{1 T}^{\perp}\left(x, \mathbf{p}_{\perp}^{2}\right) D_{1}^{h / \nu}\left(z, \mathbf{P}_{h}-z \mathbf{p}_{\perp}\right)}{2 \pi^{2} \alpha^{2} \frac{1+(1-y)^{2}}{s x y^{2}} \sum_{\nu} e_{\nu}^{2} \int d^{2} p_{\perp} f_{1}^{\nu}\left(x, \mathbf{p}_{\perp}^{2}\right) D_{1}^{h / \nu}\left(z, \mathbf{P}_{h}-z \mathbf{p}_{\perp}\right)} .
\end{aligned}
$$

In this model, the explicit form of the Sivers functions is given in Eq. (16) and the unpolarized TMDs are given in [39]. The model results for Sivers asymmetries are shown in Fig. 5 in the $\pi^{+}$and $\pi^{-}$channels and compared with the HERMES data [14] in the kinematical region

$$
\begin{aligned}
0.023<x<0.4, & 0.2<z<0.7 \\
0.31<y<0.95, & \text { and } \quad P_{h \perp}>0.05 \mathrm{GeV} .
\end{aligned}
$$

To compare with the data, $f_{1 T}^{\perp \nu}\left(x, \mathbf{p}_{\perp}^{2}\right)$ are taken at initial scale and $f_{1}^{\nu}\left(x, \mathbf{p}_{\perp}^{2}\right)$ are evolved to $\mu^{2}=2.5 \mathrm{GeV}^{2}$ following the QCD evolution [42]. For another approach of QCD evolution of TMDs, see $[65,66]$. Qualitatively our results agree with the HERMES data, but our results for the $\pi^{+}$channel are a bit smaller than the data whereas for the $\pi^{-}$channel, the model results are in good agreement with the data. This may be due to the fact that in our model, the Sivers function for the $u$-quark is smaller than the phenomenological fit (see Fig. 3). 


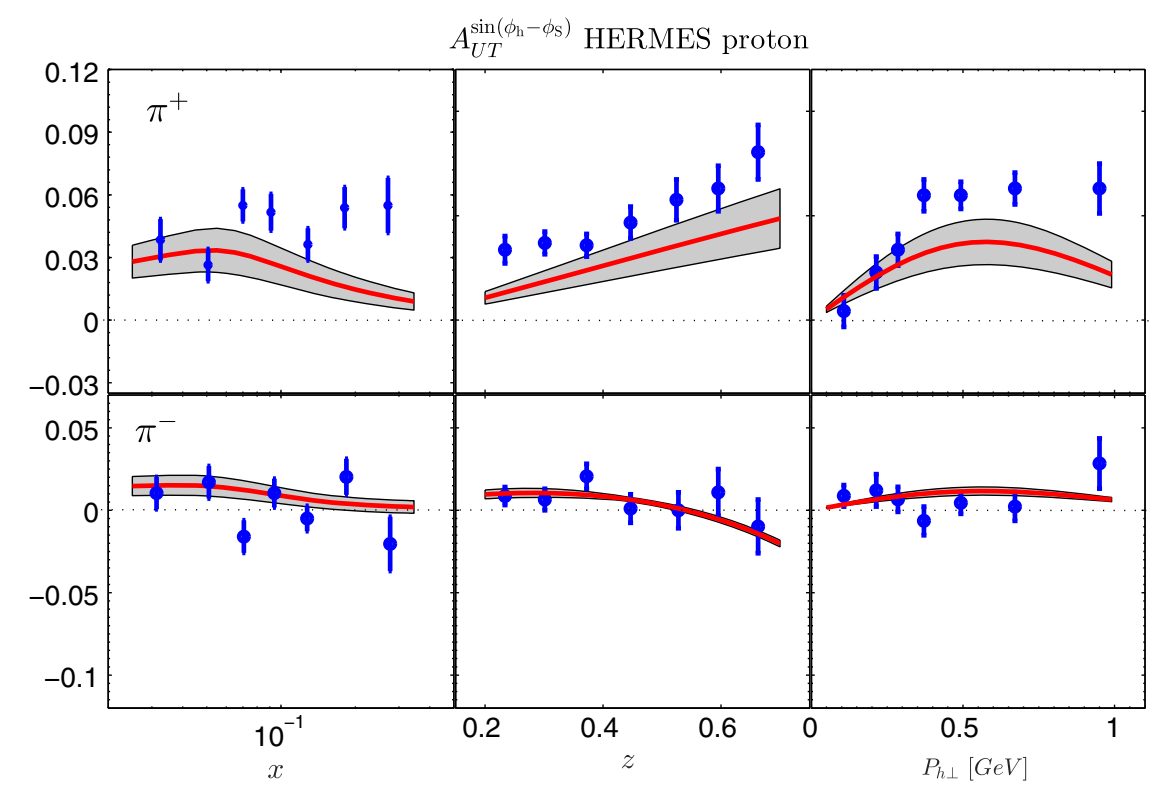

FIG. 5. Model result of Sivers asymmetries, $A_{U T}^{\sin \left(\phi_{h}-\phi_{S}\right)}$, are shown by the continuous (red) lines for $\pi^{+}$(upper row) and $\pi^{-}$(lower row) channels and compared with the HERMES data [14]. $f_{1 T}^{\perp \nu}\left(x, \mathbf{p}_{\perp}^{2}\right)$ are taken at initial scale and $f_{1}^{\nu}\left(x, \mathbf{p}_{\perp}^{2}\right)$ are evolved to $\mu^{2}=2.5 \mathrm{GeV}^{2}$ following the QCD evolution [42]. The fragmentation function $D_{1}^{h / \nu}\left(z, \mathbf{k}_{\perp}\right)$ are taken as a phenomenological [67] input at $\mu^{2}=2.5 \mathrm{GeV}^{2}$.

The Boer-Mulders asymmetry can be projected out replacing the weight factor in Eq. (28) by $\cos \left(2 \phi_{h}\right)$ and written in terms of structure functions [64] as

$$
\begin{aligned}
A_{U U}^{\cos \left(2 \phi_{h}\right)} & =\frac{4 \pi^{2} \alpha^{2} \frac{(1-y)}{s x y^{2}} F_{U U}^{\cos 2 \phi_{h}}\left(x, z, \mathbf{P}_{h \perp}\right)}{2 \pi^{2} \alpha^{2} \frac{1+(1-y)^{2}}{s x y^{2}} F_{U U}\left(x, z, \mathbf{P}_{h \perp}\right)} \\
& =\frac{4 \pi^{2} \alpha^{2} \frac{(1-y)}{s x y^{2}} \sum_{\nu} e_{\nu}^{2} \int d^{2} p_{\perp}\left\{\frac{\left(\mathbf{P}_{h \perp} \cdot \mathbf{p}_{\perp}\right)-2 z\left(\hat{\mathbf{P}}_{\perp \perp} \cdot \mathbf{p}_{\perp}\right)^{2}+z p_{\perp}^{2}}{z M_{h} M}\right\} h_{1}^{\perp \nu}\left(x, \mathbf{p}_{\perp}^{2}\right) H_{1}^{\perp \nu}\left(z,\left|\mathbf{P}_{h}-z \mathbf{p}_{\perp}\right|\right)}{2 \pi^{2} \alpha^{2} \frac{1+(1-y)^{2}}{s x y^{2}} \sum_{\nu} e_{\nu}^{2} \int d^{2} p_{\perp} f_{1}^{\nu}\left(x, \mathbf{p}_{\perp}^{2}\right) D_{1}^{h / \nu}\left(z,\left|\mathbf{P}_{h}-z \mathbf{p}_{\perp}\right|\right)} .
\end{aligned}
$$

The Boer-Mulders function in this model is given in Eq. (17). We use the unpolarized fragmentation and the Collins function $H_{1}^{\perp \nu}\left(z, \mathbf{k}_{\perp}\right)$ as phenomenological inputs $[67,68]$.

$$
\begin{gathered}
\left.D_{1}^{h / \nu}\left(z, \mathbf{k}_{\perp}\right)=D_{1}^{h / \nu}(z) \frac{e^{-\mathbf{k}_{\perp}^{2} /\left\langle k^{2} \perp\right.}}{\pi\left\langle k^{2} \perp\right.}\right\rangle \\
H_{1}^{\perp \nu}\left(z, \mathbf{k}_{\perp}\right)=\left(\frac{z M_{h}}{2 k_{\perp}}\right) 2 \mathcal{N}_{\nu}^{C}(z) D_{1}^{h / \nu}(z) h\left(k_{\perp}\right) \frac{e^{-\mathbf{k}_{\perp}^{2} /\left\langle k^{2}\right\rangle}}{\pi\left\langle k_{\perp}^{2}\right\rangle},
\end{gathered}
$$

with

$$
\begin{gathered}
\mathcal{N}_{\nu}^{C}(z)=N_{\nu}^{C} z^{\rho_{1}}(1-z)^{\rho_{2}} \frac{\left(\rho_{1}+\rho_{2}\right)^{\left(\rho_{1}+\rho_{2}\right)}}{\rho_{1}^{\rho_{1}} \rho_{2}^{\rho_{2}}}, \\
h\left(k_{\perp}\right)=\sqrt{2 e} \frac{k_{\perp}}{M_{h}} e^{-\mathbf{k}_{\perp}^{2} / M_{h}^{2}}
\end{gathered}
$$

where $z=P_{h}^{-} / k^{-}$is the energy fraction carried by the fragmenting quark of momentum $\mathbf{k}$. The values of the parameters are listed in [68] and $D_{1}^{h / \nu}(z)$ is taken from the phenomenological extraction [67]. The Boer-Mulders asymmetry is shown in Fig. 6. We compare our model results with the HERMES data $[26,69]$ in the kinematical region

$$
\begin{aligned}
0.023 & <x<1.0, \quad 0.2<z<1.0, \\
0.3 & <y<0.85, \quad \text { and } \quad P_{h \perp}>0.05 \mathrm{GeV} .
\end{aligned}
$$

The Boer-Mulders asymmetries agree with the HERMES data within error bars, except the plot with respect to $z$ in the $\pi^{-}$channel (Fig. 6). The $A_{U U}^{\cos \left(2 \phi_{h}\right)}$ asymmetry gets a twist- 4 contribution due to the Cahn effect [26] which is not included here.

Similarly, $\cos \left(\phi_{h}\right)$-weighted asymmetry also receives contribution from the Cahn effect and Boer-Mulders function and is defined [64] by 


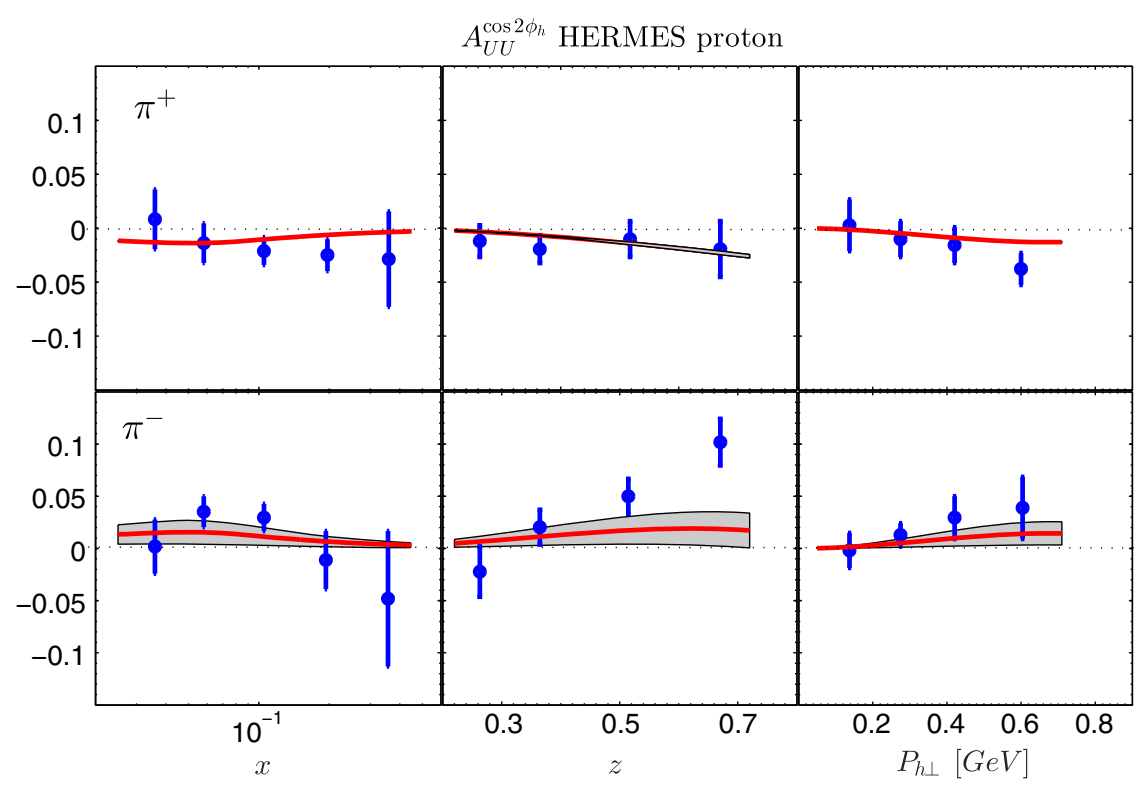

FIG. 6. Model result of Boer-Mulders asymmetries, $A_{U U}^{\cos 2 \phi_{h}}$. The continuous (red) lines represent the model results and the data are measured by the HERMES Collaboration [26,69]. $h_{1}^{\perp \nu}\left(x, \mathbf{p}_{\perp}^{2}\right)$ are taken at initial scale and $f_{1}^{\nu}\left(x, \mathbf{p}_{\perp}^{2}\right)$ are evolved at $\mu^{2}=2.5 \mathrm{GeV}^{2}$ following the QCD evolution [42]. The fragmentation function $H_{1}^{\perp \nu}\left(z, \mathbf{k}_{\perp}\right)$ are taken as a phenomenological [68,70] input at $\mu^{2}=2.5 \mathrm{GeV}^{2}$.

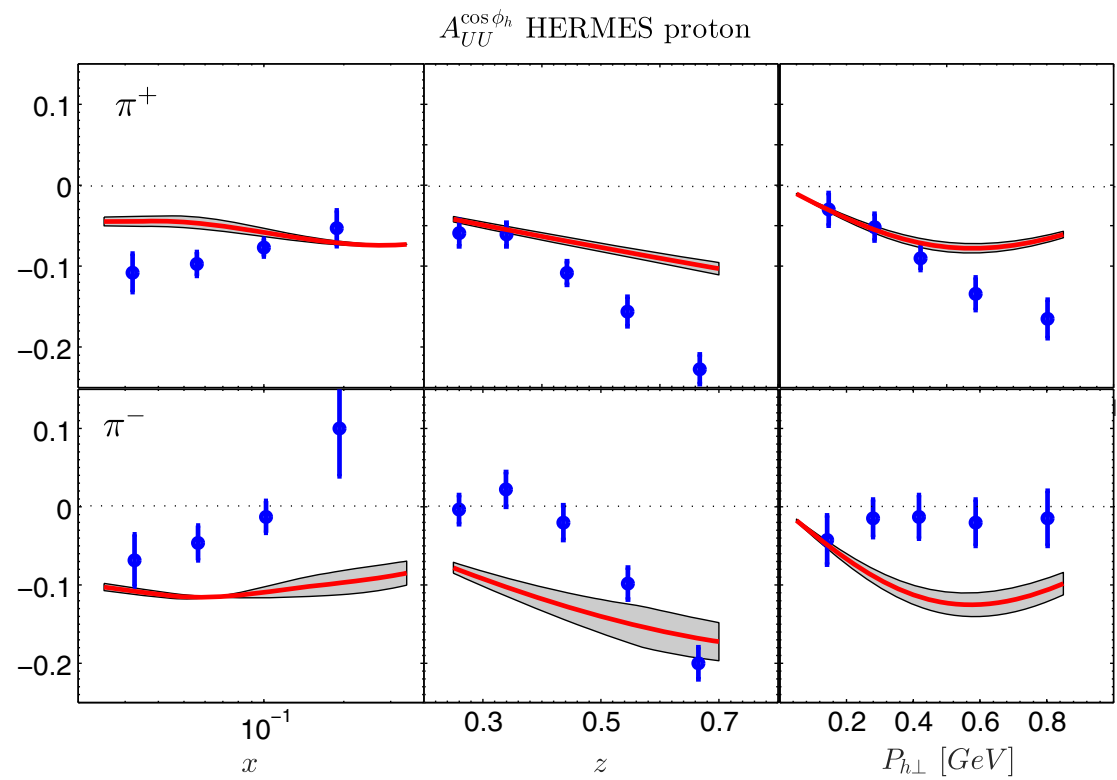

FIG. 7. Model results for $A_{U U}^{\cos \phi_{h}}$ are shown by the continuous (red) lines and compared with HERMES data [71]. $h_{1}^{\perp \nu}\left(x, \mathbf{p}_{\perp}\right)$ are taken at initial scale and $f_{1}^{\nu}\left(x, \mathbf{p}_{\perp}\right)$ are evolved at $\mu^{2}=2.5 \mathrm{GeV}^{2}$ following the QCD evolution [42]. The fragmentation function $H_{1}^{\perp \nu}\left(z, \mathbf{k}_{\perp}\right)$ are taken as a phenomenological $[68,70]$ input at $\mu^{2}=2.5 \mathrm{GeV}^{2}$.

$$
\begin{aligned}
A_{U U}^{\cos \phi_{h}} & =\frac{4 \pi^{2} \alpha^{2} \frac{(2-y) \sqrt{(1-y)}}{s x y^{2}} F_{U U}^{\cos \phi_{h}}\left(x, z, \mathbf{P}_{h \perp}\right)}{2 \pi^{2} \alpha^{2} \frac{1+(1-y)^{2}}{s x y^{2}} F_{U U}\left(x, z, \mathbf{P}_{h \perp}\right)} \\
& =\frac{4 \pi^{2} \alpha^{2} \frac{(1-y)}{s x y^{2}}\left(-\frac{2}{Q}\right) \sum_{\nu} e_{\nu}^{2} \int d^{2} p_{\perp}\left[\left(\hat{\mathbf{P}}_{h \perp} \cdot \mathbf{p}_{\perp}\right) f_{1}^{\nu} D_{1}^{h / \nu}+\frac{p_{\perp}^{2}\left(P_{h \perp}-z \hat{\mathbf{P}}_{h \perp} \cdot \mathbf{p}_{\perp}\right)}{z M_{h} M} h_{1}^{\perp \nu} H_{1}^{\perp \nu}\right]}{2 \pi^{2} \alpha^{2} \frac{1+(1-y)^{2}}{s x y^{2}} \sum_{\nu} e_{\nu}^{2} \int d^{2} p_{\perp} f_{1}^{\nu}\left(x, \mathbf{p}_{\perp}^{2}\right) D_{1}^{h / \nu}\left(z,\left|\mathbf{P}_{h}-z \mathbf{p}_{\perp}\right|\right)} .
\end{aligned}
$$


The model result for the asymmetry $A_{U U}^{\cos \left(\phi_{h}\right)}$ is shown in Fig. 7 for the $\pi^{+}$and $\pi^{-}$channels and compared with the HERMES data [71]. Agreement of our result with the data is not so good. The asymmetry involves the higher twist effect (Cahn effect). Our simple model is not suitable to describe the higher twist effects. This might be the reason for this discrepancy with the data. Recently, the BoerMulders function and the Cahn effects have been extracted from the experimental data of $\cos 2 \phi_{h}$ and $\cos \phi_{h}$ weighted asymmetries [72].

\section{SPIN DENSITIES}

The spin density of unpolarized quarks with flavor $\nu$ in a transversely polarized proton is defined [73] as

$f_{\nu / P^{\uparrow}}\left(x, \mathbf{p}_{\perp}\right)=f_{1}^{\nu}\left(x, \mathbf{p}_{\perp}^{2}\right)-\frac{\mathbf{S} .\left(\hat{\mathbf{P}} \times \mathbf{p}_{\perp}\right)}{M} f_{1 T}^{\perp \nu}\left(x, \mathbf{p}_{\perp}^{2}\right)$.

Here for the SIDIS process, we take $\hat{\mathbf{P}}$ as being along the direction of the momentum transfer $\hat{z}$-axis and the spin of the proton $\mathbf{S}$ is along $\hat{\mathbf{y}}$. The spin density $f_{\nu / p^{\uparrow}}\left(x, \mathbf{p}_{\perp}\right)$ in the transverse momentum plane are shown in Fig. 8 for both $u$ and $d$ quarks, where the longitudinal momentum fraction $x=0.2$. The distributions are not symmetric, but rather distorted towards the left for the $u$ quark and towards the right for the $d$ quark. This left-right distortion in the distribution was observed for the first time by D. Sivers [5] and can be explained by the nonvanishing Sivers function $f_{1 T}^{\perp \nu}\left(x, \mathbf{p}_{\perp}^{2}\right)$. This is known as the Sivers effect, where the quarks in a transversely polarized target have a transverse momentum asymmetry in the perpendicular direction to the nucleon spin $\mathbf{S}$. The left distortion is due to the negative distribution of the Sivers functions for the $u$ quark and the right distortion is due to the positive distribution of the Sivers function for the $d$ quark. Similar kinds of distortions are observed in other model calculations [44] as well as in lattice QCD [74].

Similarly, the spin density for transversely polarized quarks with flavor $\nu$ in an unpolarized proton is defined [73] as

$f_{\nu^{\uparrow} / P}\left(x, \mathbf{p}_{\perp}\right)=\frac{1}{2}\left[f_{1}^{\nu}\left(x, \mathbf{p}_{\perp}^{2}\right)-\frac{\mathbf{s} .\left(\hat{\mathbf{P}} \times \mathbf{p}_{\perp}\right)}{M} h_{1}^{\perp \nu}\left(x, \mathbf{p}_{\perp}^{2}\right)\right]$,

where $\mathbf{s}$ is the spin of the interior quark. The spin density $f_{\nu^{\uparrow} / P}\left(x, \mathbf{p}_{\perp}\right)$ is shown in Fig. 9 for quark spin $\mathbf{s}$ along $\hat{\mathbf{y}}$ with $x=0.2$. Since Boer-Mulders functions are negative for both $u$ and $d$ quarks, we observed only a left shift, unlike the Sivers effect, as obtained in Fig. 9.

The Sivers function is related with the anomalous magnetic moment and the orbital angular momentum of partons. The Pauli form factor defined by the correlator with helicity-flip vector current is written in terms of overlap representations as

$$
\begin{aligned}
F_{2}^{\nu}\left(Q^{2}\right)= & -\frac{2 M}{\left(q^{1}-i q^{2}\right)} \int_{0}^{1} \frac{d x d^{2} p_{\perp}}{16 \pi^{3}} \\
& \times\left[C_{S}^{2} \sum_{\lambda_{q}} \sum_{\lambda_{N} \neq \lambda_{N}^{\prime}} \psi_{\lambda_{q}}^{\lambda_{N}^{\dagger}}\left(x, \mathbf{p}_{\perp}\right) \psi_{\lambda_{q}}^{\lambda_{N}^{\prime}}\left(x, \mathbf{p}_{\perp}\right)\right. \\
& \left.+C_{A}^{2} \sum_{\lambda_{q}} \sum_{\lambda_{D}} \sum_{\lambda_{N} \neq \lambda_{N}^{\prime}} \psi_{\lambda_{q} \lambda_{D}}^{\lambda_{N} \dagger}\left(x, \mathbf{p}_{\perp}\right) \psi_{\lambda_{q} \lambda_{D}}^{\lambda_{N}^{\prime}}\left(x, \mathbf{p}_{\perp}\right)\right]
\end{aligned}
$$

$=\int_{0}^{1} d x\left(C_{S}^{2} N_{S}^{\nu 2}-C_{A}^{2} \frac{1}{3} N_{0}^{\nu 2}\right) 2 T_{3}^{\nu}(x)(1-x)^{3} e^{-Q^{2 \frac{\ln (1 / x)}{4 x^{2}}},}$

where $T_{3}^{\nu}(x)=x^{a_{1}^{\nu}+a_{2}^{\nu}-1}(1-x)^{b_{1}^{\nu}+b_{2}^{\nu}-1}$. The anomalous magnetic moment $\kappa^{\nu}$ can be found from the Pauli form factor in the limit $Q^{2}=0, \kappa^{\nu}=F_{2}^{\nu}(0)$. Thus

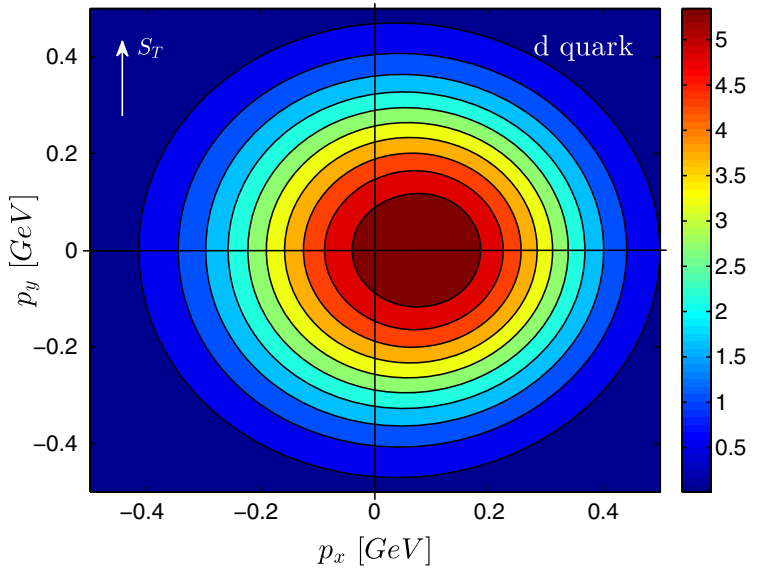

FIG. 8. Spin density $f_{\nu / P^{\uparrow}}\left(x, \mathbf{p}_{\perp}\right)$ [Eq. (43)] are shown in the transverse momentum plane for $u$ and $d$ quarks with $x=0.2$. The proton spin $\mathbf{S}$ is along the $y$-axis and the momentum of the proton $\mathbf{P}$ is along the $z$-direction. 

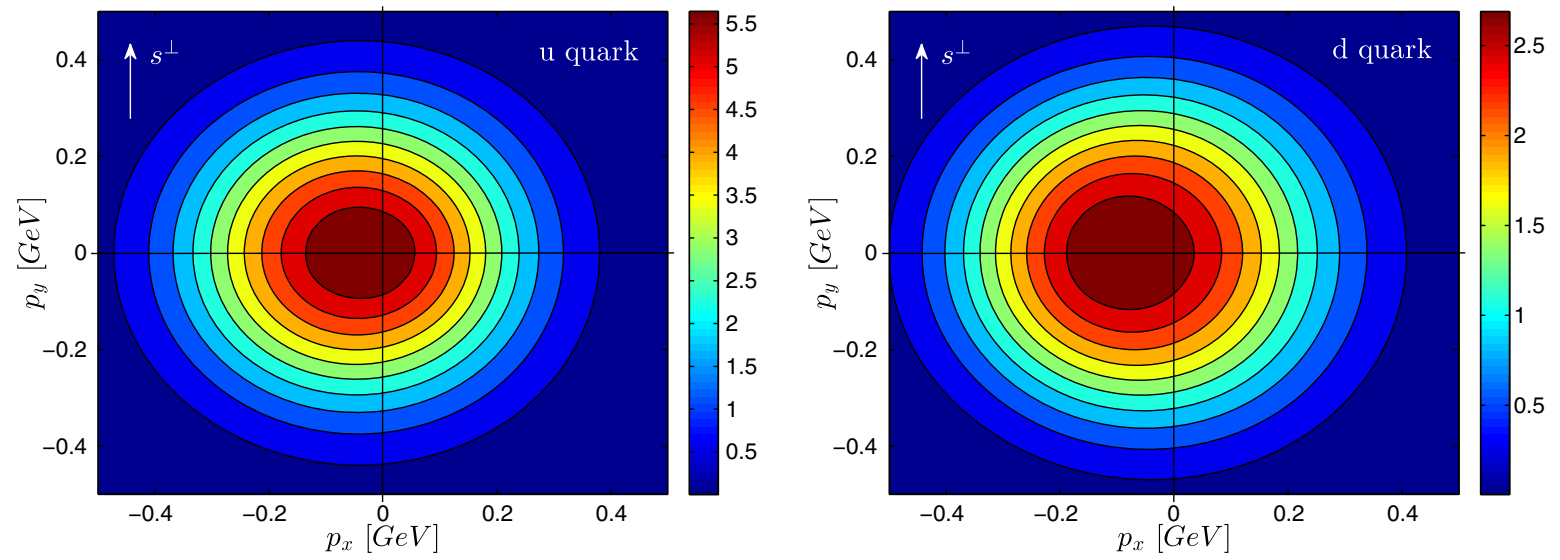

FIG. 9. Spin density $f_{\nu^{\uparrow} / P}\left(x, \mathbf{p}_{\perp}\right)$ [Eq. (44)] are shown in the transverse momentum plane for $u$ and $d$ quarks with $x=0.2$. The quark spin $\mathbf{S}$ is along the $y$-axis and the momentum of the proton $\mathbf{P}$ is along the $z$-direction.

$$
\begin{aligned}
\kappa^{\nu} & =\int_{0}^{1} d x \kappa^{\nu}(x) \\
& =\int_{0}^{1} d x\left(C_{S}^{2} N_{S}^{\nu 2}-C_{A}^{2} \frac{1}{3} N_{0}^{\nu 2}\right) 2 T_{3}^{\nu}(x)(1-x)^{3} .
\end{aligned}
$$

A simple relation between the integrated Sivers function (over $\mathbf{p}_{\perp}$ ) and anomalous magnetic moments is found as

$$
f_{1 T}^{\perp \nu}(x)=-C_{F} \alpha_{s} \mathcal{G}^{\nu}(x) \kappa^{\nu}(x) .
$$

In this model, the relation cannot be derived analytically; however numerical calculation gives the lensing function as

$$
\left.\mathcal{G}^{\nu}(x) \simeq \frac{1}{4(1-x)}\right|_{\nu=u, d} .
$$

A similar type of lensing function is found in [75]. In Ref. [76], $\mathcal{G}^{\nu}(x) \propto 1 /(1-x)^{\eta}$ where $\eta$ is typically around 0.4 but $\eta$ can vary between 0.03 and 2 .

The total longitudinal angular momentum of parton $\nu$ is defined in terms of the moment of the GPDs as

$$
J^{\nu}=\frac{1}{2} \int_{0}^{1} d x x\left[H^{\nu}(x, 0,0)+E^{\nu}(x, 0,0)\right] .
$$

In the forward limit, the moment of the $E$ and $H$ GPDs satisfies

$$
\begin{gathered}
\int_{0}^{1} d x H^{\nu}(x, 0,0)=n^{\nu}=\int_{0}^{1} d x d^{2} \mathbf{p}_{\perp} f_{1}^{\nu}\left(x, \mathbf{p}_{\perp}^{2}\right) \\
\int_{0}^{1} d x E^{\nu}(x, 0,0)=\kappa^{\nu}
\end{gathered}
$$

where $n^{u}=2$ and $n^{d}=1$ for the proton. From isospin symmetry flavored anomalous magnetic moments are $\kappa^{u}=$ 1.673 and $\kappa^{d}=-2.033$. GPDs are discussed in this model
[40]. We define $\kappa^{\nu}=\int d x \kappa^{\nu}(x)$ and $\kappa^{\nu}(x)=E^{\nu}(x, 0,0)$. Therefore, Eq. (48) is modified as

$$
f_{1 T}^{\perp \nu}(x) \simeq-C_{F} \alpha_{s} \frac{1}{4(1-x)} E^{\nu}(x, 0,0) .
$$

Thus the longitudinal angular momentum can be calculated from the moment of the Sivers function and unpolarized TMDs as

$$
J^{\nu}=\frac{1}{2} \int_{0}^{1} d x x\left[f_{1}^{\nu}(x)-\frac{4(1-x)}{C_{F} \alpha_{s}} f_{1 T}^{\perp \nu}(x)\right] .
$$

In this model, we obtain

$$
J^{u}=0.9559 \quad \text { and } \quad J^{d}=-0.5791 .
$$

Total contribution to the nucleon spin from $u$ and $d$ quarks is 0.3768 at the initial scale $\mu_{0}=0.8 \mathrm{GeV}$. The cloudy bag model [77] and lattice calculations predict total angular momentum contribution about 0.24 at a scale of $\mu^{2}=4 \mathrm{GeV}^{2}$.

\section{CONCLUSIONS}

We have presented the results for $T$-odd TMDs, namely, the Sivers and Boer-Mulders functions, in a light-front quark-diquark model of the proton and the spin asymmetries in SIDIS associated with these functions. It is well known that the final-state interaction is responsible for producing the required complex phase in the amplitude which gives rise to the Sivers asymmetries. Though the proton wave function in principle cannot describe FSI (as the participating quark comes out of the proton state), as Hwang [47] proposed, we have modeled the LFWFs to incorporate the effects of the FSI. This is done by extending the wave functions in the quark-diquark model to have complex phases consistent with the SIDIS amplitudes. The 
complex phases in the LFWFs produce the Sivers and Boer-Mulders functions. Both the Sivers and Boer Mulders functions and their moments are evaluated in this model and compared with other model and phenomenological fits. The Sivers asymmetry $A_{U T}^{\sin \left(\phi_{h}-\phi_{S}\right)}$ for the $\pi^{+}$channel is found to be a bit smaller than the experimental data; better agreements are observed for the Boer-Mulders asymmetry $A_{U U}^{\cos \left(2 \phi_{h}\right)}$ for both $\pi^{+}$and $\pi^{-}$ channels. Sivers and Boer-Mulders functions help us to understand the spin structure of the proton at the parton level. Due to the Sivers effect the spin density of an unpolarized quark in a transversely polarized proton is found to be asymmetric in the perpendicular direction to the nuclear spin. The distortions due to the Sivers effect in our model for both $u$ and $d$ quarks are consistent with the results found in other models and lattice QCD. Since the Sivers function is negative for $u$ and positive for the $d$ quark, the distortion for the $u$ quark is in the opposite direction of the $d$ quark. Similarly, the Boer-Mulders function produces the distortion in the spin density of a transversely polarized quark in a transversely polarized proton. Since the Boer-Mulders function has the same sign for both $u$ and $d$ quarks, the distortions in the spin densities are also in the same direction. The Sivers function integrated over the transverse momentum is related to the anomalous magnetic moment through the lensing function. Our model predicts that the lensing function should go as $(1-x)^{-1}$.
[1] M. Anselmino, A. Efremov, and E. Leader, Phys. Rep. 261, 1 (1995); M. Anselmino, A. Efremov, and E. Leader, Phys. Rep. 281, 399(E) (1997).

[2] V. Barone, A. Drago, and P. G. Ratcliffe, Phys. Rep. 359, 1 (2002).

[3] D. L. Adams et al. (E581 and E704 Collaborations), Phys. Lett. B 261, 201 (1991).

[4] D. L. Adams et al. (FNAL-E704 Collaboration), Phys. Lett. B 264, 462 (1991).

[5] D. W. Sivers, Phys. Rev. D 41, 83 (1990).

[6] D. Boer and P. J. Mulders, Phys. Rev. D 57, 5780 (1998).

[7] S. J. Brodsky, D. S. Hwang, and I. Schmidt, Nucl. Phys. B642, 344 (2002).

[8] J. C. Collins, Phys. Lett. B 536, 43 (2002).

[9] D. Boer, P. J. Mulders, and F. Pijlman, Nucl. Phys. B667, 201 (2003).

[10] A. V. Belitsky, X. Ji, and F. Yuan, Nucl. Phys. B656, 165 (2003).

[11] C. J. Bomhof, P. J. Mulders, and F. Pijlman, Phys. Lett. B 596, 277 (2004).

[12] M. G. Alekseev et al. (COMPASS Collaboration), Phys. Lett. B 692, 240 (2010).

[13] M. Alekseev et al. (COMPASS Collaboration), Phys. Lett. B 673, 127 (2009).

[14] A. Airapetian et al. (HERMES Collaboration), Phys. Rev. Lett. 103, 152002 (2009).

[15] X. Qian et al. (Jefferson Lab Hall A Collaboration), Phys. Rev. Lett. 107, 072003 (2011).

[16] G. Sbrizzai (COMPASS Collaboration), Int. J. Mod. Phys. Conf. Ser. 40, 1660032 (2016).

[17] L. Adamczyk et al. (STAR Collaboration), Phys. Rev. Lett. 116, 132301 (2016).

[18] U. D’Alesio and F. Murgia, Phys. Rev. D 70, 074009 (2004).

[19] A. V. Efremov, K. Goeke, S. Menzel, A. Metz, and P. Schweitzer, Phys. Lett. B 612, 233 (2005).

[20] M. Anselmino, M. Boglione, U. D’Alesio, A. Kotzinian, F. Murgia, and A. Prokudin, Phys. Rev. D 72, 094007 (2005); 72, 099903(E) (2005).
[21] J. C. Collins, A. V. Efremov, K. Goeke, M. Grosse Perdekamp, S. Menzel, B. Meredith, A. Metz, and P. Schweitzer, Phys. Rev. D 73, 094023 (2006).

[22] M. Anselmino, M. Boglione, U. D'Alesio, A. Kotzinian, S. Melis, F. Murgia, A. Prokudin, and C. Turk, Eur. Phys. J. A 39, 89 (2009).

[23] M. Anselmino, M. Boglione, U. D’Alesio, S. Melis, F. Murgia, and A. Prokudin, Phys. Rev. D 88, 054023 (2013).

[24] M. Anselmino, M. Boglione, U. D’Alesio, F. Murgia, and A. Prokudin, J. High Energy Phys. 04 (2017) 046.

[25] A. Martin, F. Bradamante, and V. Barone, Phys. Rev. D 95, 094024 (2017).

[26] V. Barone, S. Melis, and A. Prokudin, Phys. Rev. D 81, 114026 (2010).

[27] B. Zhang, Z. Lu, B. Q. Ma, and I. Schmidt, Phys. Rev. D 77, 054011 (2008).

[28] B. U. Musch, P. Hagler, M. Engelhardt, J. W. Negele, and A. Schafer, Phys. Rev. D 85, 094510 (2012).

[29] L. P. Gamberg, G. R. Goldstein, and M. Schlegel, Phys. Rev. D 77, 094016 (2008).

[30] M. Burkardt and B. Hannafious, Phys. Lett. B 658, 130 (2008).

[31] B. Pasquini and F. Yuan, Phys. Rev. D 81, 114013 (2010).

[32] F. Yuan, Phys. Lett. B 575, 45 (2003).

[33] A. Bacchetta, A. Schaefer, and J. J. Yang, Phys. Lett. B 578, 109 (2004).

[34] A. Courtoy, F. Fratini, S. Scopetta, and V. Vento, Phys. Rev. D 78, 034002 (2008).

[35] M. Burkardt, Phys. Rev. D 69, 057501 (2004).

[36] S. Meissner, A. Metz, and K. Goeke, Phys. Rev. D 76, 034002 (2007).

[37] T. Maji and D. Chakrabarti, Phys. Rev. D 94, 094020 (2016).

[38] D. Chakrabarti, T. Maji, C. Mondal, and A. Mukherjee, Phys. Rev. D 95, 074028 (2017).

[39] T. Maji and D. Chakrabarti, Phys. Rev. D 95, 074009 (2017).

[40] T. Maji, C. Mondal, and D. Chakrabarti, Phys. Rev. D 96, 013006 (2017). 
[41] T. Maji, D. Chakrabarti, and O. V. Teryaev, Phys. Rev. D 96, 114023 (2017).

[42] S. M. Aybat and T. C. Rogers, Phys. Rev. D 83, 114042 (2011).

[43] R. Jakob, P. J. Mulders, and J. Rodrigues, Nucl. Phys. A626, 937 (1997).

[44] A. Bacchetta, F. Conti, and M. Radici, Phys. Rev. D 78, 074010 (2008).

[45] J. R. Ellis, D. S. Hwang, and A. Kotzinian, Phys. Rev. D 80, 074033 (2009).

[46] S. J. Brodsky, D. S. Hwang, and I. Schmidt, Phys. Lett. B 530, 99 (2002).

[47] D. S. Hwang, J. Korean Phys. Soc. 62, 581 (2013).

[48] T. Gutsche, V. E. Lyubovitskij, I. Schmidt, and A. Vega, Phys. Rev. D 89, 054033 (2014); 92, 019902(E) (2015).

[49] S. J. Brodsky and G. F. de Teramond, Phys. Rev. D 77, 056007 (2008).

[50] D. Chakrabarti and C. Mondal, Phys. Rev. D 88, 073006 (2013).

[51] M. Burkardt, Phys. Rev. D 69, 091501 (2004).

[52] K. Goeke, S. Meissner, A. Metz, and M. Schlegel, Phys. Lett. B 637, 241 (2006).

[53] M. Anselmino, M. Boglione, and S. Melis, Phys. Rev. D 86, 014028 (2012).

[54] M. Anselmino, M. Boglione, U. D'Alesio, S. Melis, F. Murgia, and A. Prokudin, arXiv:1107.4446.

[55] X. Ji, J. W. Qiu, W. Vogelsang, and F. Yuan, Phys. Lett. B 638, 178 (2006).

[56] M. Anselmino, M. Boglione, A. Prokudin, and C. Turk, Eur. Phys. J. A 31, 373 (2007).

[57] X. Ji, J. P. Ma, and F. Yuan, Phys. Rev. D 71, 034005 (2005).

[58] X. Ji, J. P. Ma, and F. Yuan, Phys. Lett. B 597, 299 (2004).

[59] J. Collins, in Foundations of Perturbative QCD, Cambridge Monographs on Particle Physics, Nuclear Physics, and Cosmology Vol. 32 (Cambridge University Press, Cambridge, England, 2011).

[60] M. G. Echevarria, A. Idilbi, and I. Scimemi, J. High Energy Phys. 07 (2012) 002.
[61] M. G. Echevarra, A. Idilbi, and I. Scimemi, Phys. Lett. B 726, 795 (2013).

[62] S. M. Aybat, J. C. Collins, J. W. Qiu, and T. C. Rogers, Phys. Rev. D 85, 034043 (2012).

[63] S. M. Aybat, A. Prokudin, and T. C. Rogers, Phys. Rev. Lett. 108, 242003 (2012).

[64] M. Anselmino, M. Boglione, U. D'Alesio, S. Melis, F. Murgia, E. R. Nocera, and A. Prokudin, Phys. Rev. D 83, 114019 (2011).

[65] M. G. Echevarria, A. Idilbi, Z. B. Kang, and I. Vitev, Phys. Rev. D 89, 074013 (2014).

[66] M. G. Echevarria, A. Idilbi, A. Schfer, and I. Scimemi, Eur. Phys. J. C 73, 2636 (2013).

[67] S. Kretzer, E. Leader, and E. Christova, Eur. Phys. J. C 22, 269 (2001).

[68] M. Anselmino, M. Boglione, U. D’Alesio, S. Melis, F. Murgia, and A. Prokudin, Phys. Rev. D 87, 094019 (2013).

[69] F. Giordano, R. Lamb, D. G. Crabb, Y. Prok, M. Poelker, S. Liuti, D. B. Day, and X. Zheng (HERMES Collaboration), AIP Conf. Proc. 1149, 423 (2009).

[70] M. Anselmino, M. Boglione, U. D’Alesio, A. Kotzinian, F. Murgia, A. Prokudin, and C. Turk, Phys. Rev. D 75, 054032 (2007).

[71] A. Airapetian et al. (HERMES Collaboration), Phys. Rev. D 87, 012010 (2013).

[72] E. Christova, E. Leader, and M. Stoilov, arXiv:1705.10613.

[73] A. Bacchetta, U. D'Alesio, M. Diehl, and C. A. Miller, Phys. Rev. D 70, 117504 (2004).

[74] M. Gockeler, Ph. Hägler, R. Horsley, Y. Nakamura, D. Pleiter, P. E. L. Rakow, A. Schäfer, G. Schierholz, H. Stüben, and J. M. Zanotti (QCDSF and UKQCD Collaborations), Phys. Rev. Lett. 98, 222001 (2007).

[75] Z. Lu and I. Schmidt, Phys. Rev. D 75, 073008 (2007).

[76] A. Bacchetta and M. Radici, Phys. Rev. Lett. 107, 212001 (2011).

[77] C. A. Aidala, S. D. Bass, D. Hasch, and G. K. Mallot, Rev. Mod. Phys. 85, 655 (2013). 